\title{
Mucosite oral em crianças com câncer - revisão de literatura
}

\author{
Oral mucositis in children with cancer - literature review
}

\author{
Isabel Nemoto Vergara Sasada* \\ Maria Cristina Munerato** \\ Lauro Jose Gregianin ${ }^{* * *}$
}

\section{Resumo}

Mucosite oral (MO) é caracterizada pela inflamação da mucosa bucal frequentemente observada após utilização de agentes quimioterápicos e da radioterapia na região de cabeça e pescoço, variando desde eritema localizado até úlceras extensas, podendo ser agravada pela saúde bucal inadequada. Objetivo: o presente estudo objetiva realizar uma revisão de literatura acerca da MO. Revisão de literatura: a MO representa um alto risco de infecção, podendo evoluir para infecções sistêmicas graves, interferindo no tratamento e na sobrevida do paciente. O conhecimento da etiologia e dos mecanismos de prevenção e tratamento é importante para diminuir os riscos associados a infecções oportunistas, dificuldade de alimentação e septicemia. A MO é um processo complexo e multifatorial, envolvendo todos os tecidos e elementos celulares da mucosa oral. Novas evidências sugerem que o risco de desenvolvimento da mucosite oral pode ser influenciado por fatores genéticos. As abordagens têm sido constantemente avaliadas, buscando a prevenção, o tratamento, a diminuição dos riscos e a melhora na qualidade de vida dos pacientes. Considerações finais: condições de saúde bucal prévia adequadas e acompanhamento odontológico durante o tratamento oncológico são importantes na prevenção das complicações estomatológicas. O tratamento preventivo e paliativo da MO auxilia na prevenção de infecções secundárias e é recomendado para alívio dos sintomas.

Palavras-chave: Mucosite oral. Quimioterapia. Radioterapia. Saúde bucal.

\section{Introdução}

A cavidade bucal é revestida pela membrana mucosa, que é o forramento úmido que cobre tanto o trato gastrointestinal como as vias aéreas e as outras cavidades do organismo que se comunicam com o ambiente externo. A mucosa bucal é composta por epitélio pavimentoso estratificado, queratinizado ou não, cobrindo o tecido conjuntivo.

Suas funções são de proteção, sensação, secreção e regulação térmica. É classificada em três tipos: 1) mastigatória, que cobre as áreas expostas às forças compressivas e ao atrito da mastigação, 2) mucosa de revestimento e 3) mucosa especializada da superfície dorsal da língua ${ }^{1,2}$.

A mucosite oral $(\mathrm{MO})^{4}$ pode ser definida como uma alteração da mucosa de revestimento da cavidade bucal que pode ocorrer durante o tratamento oncológico. Segundo Neville et al. $^{3}$ (2009), o dano mucoso secundário ao tratamento antineoplásico é complexo e parece surgir de uma série de eventos celulares e moleculares que ocorrem não só em epitélio como também no estroma subjacente. Diferenças genéticas na taxa de apoptose tecidual, lesões microvasculares a partir da apoptose do endotélio e níveis séricos elevados de fator de necrose tumoral alfa e interleucina-6 parecem estar envolvidos.

Odontopediatra, mestranda em Saúde da Criança e do Adolescente pela Faculdade de Medicina da Universidade Federal do Rio Grande do Sul, Porto Alegre, Rio Grande do Sul, Brasil.

Doutora em Estomatologia, Unidade de Estomatologia do Hospital de Clínicas de Porto Alegre, Universidade Federal do Rio Grande do Sul, Porto Alegre, Rio Grande do Sul, Brasil.

... Doutor em Saúde da Criança e do Adolescente pela Faculdade de Medicina da Universidade Federal do Rio Grande do Sul, Porto Alegre, Rio Grande do Sul, Brasil. 
Além dos efeitos diretos dos agentes antineoplási$\cos$, fatores de risco adicionais incluem idade precoce, higiene bucal deficiente, focos de infecção bucal, má nutrição, função salivar deficiente, uso de tabaco e/ou álcool.

Atualmente, alguns autores sugerem o termo "mucosite do trato alimentar", visto que as alterações bucais constituem apenas parte de uma série de danos que ocorrem em todo o trato gastrointestinal ${ }^{4,5}$. A mucosite associada à quimioterapia envolve, comumente, as superfícies não ceratinizadas da mucosa jugal, superfície ventrolateral da língua, palato mole e assoalho bucal. A radioterapia afeta, sobretudo, as superfícies mucosas atingidas diretamente pelo foco da radiação $0^{3,4}$.

Portanto, a MO, usualmente, apresenta uma etiologia multifatorial resultante da toxicidade direta dos agentes quimioterápicos e/ou da toxicidade indireta pela neutropenia ${ }^{6}$.

A MO induzida por radioterapia (RT) acomete praticamente todos os pacientes submetidos à radiação na região de cabeça e pescoço. A associação entre RT e quimioterapia (QT) determina um impacto importante sobre a mucosa oral, sendo relatada $\mathrm{MO}$ em $89 \%$ dos pacientes que receberam ambas as modalidades de tratamento, contra $22 \%$ dos pacientes que receberam QT exclusivamente ${ }^{7,8}$.

Pacientes mais jovens tendem a desenvolver MO mais intensa que pacientes mais velhos, quando tratados com o mesmo protocolo de QT. Isso ocorre, provavelmente, pela velocidade de divisão celular nas crianças, mas também favorece a recuperação do grupo jovem em menor tempo ${ }^{9,10}$.

A condição dental, o desequilíbrio da microbiota bucal causada por acúmulo de biofilme ou problemas periodontais, o consumo de bebidas alcoólicas e o fumo são fatores que interferem na progressão da mucosite ${ }^{4,5}$. Outro fator agravante é a suscetibilidade às infecções oportunistas por microrganismos normalmente presentes na cavidade bucal, que invadem os tecidos lesados e, quando associados à neutropenia, aumentam o risco de septicemia ${ }^{11}$. Alterações das glândulas salivares que determinam hipossalivação podem acentuar as lesões, tanto pela diminuição da lubrificação da mucosa quanto pela alteração do padrão antimicrobiano da saliva. O uso de próteses ou aparelhos ortodônticos deve ser avaliado individualmente pelo risco de traumatismos ${ }^{4}$.

\section{Revisão de literatura}

\section{Etiologia da MO}

A MO induzida por QT ocorre por dois mecanismos distintos: a toxicidade direta da QT sobre a mucosa e a mielossupressão gerada pelo tratamen- to. Sua patogênese está ligada à renovação celular diminuída nas camadas basais do epitélio, que se torna incapaz de repor satisfatoriamente as células descamadas. Células da orofaringe, dos epitélios bucal e intestinal, bem como da medula óssea dividem-se rapidamente e são mais sensíveis aos efeitos da terapia antineoplásica ${ }^{7,12}$. Essa interferência na manutenção da espessura epitelial da mucosa bucal resulta em inflamação, atrofia e ulceração localizada ou difusa. Paralelamente, a QT altera, também, a flora microbiana da cavidade bucal, a quantidade e composição da saliva e a maturação epitelial ${ }^{7,13}$. A barreira mucosa comprometida representa um fator de risco maior para morbidade e até mortalidade nos pacientes oncológicos imunossuprimidos ${ }^{7,14}$.

A manifestação clínica mais precoce na mucosa é o desenvolvimento de uma coloração esbranquiçada pela ausência de descamação suficiente da ceratina. Isso logo é seguido pela perda dessa camada e por sua substituição pela mucosa atrófica, a qual é edemaciada, eritematosa e friável. Subsequentemente, áreas de ulceração desenvolvem-se com a formação de uma membrana superficial fibrinopurulenta amarelada e removível. Dor, ardência e desconforto são sintomas que podem se acentuar durante a alimentação ou a higiene bucal ${ }^{3,4}$.

Segundo Adamietz et al..$^{15}$ (1998), a origem das lesões mucosas induzidas pelo tratamento oncológico é de natureza biológica ou tóxica, apesar de o desenvolvimento posterior ser estimulado por infecções oportunistas, que são agravadas pelo desequilíbrio do sistema imunológico. Danos às glândulas salivares causam redução do fluxo salivar, bem como alterações na composição da saliva e de seu $\mathrm{pH}$, que são seguidos por mudanças na microflora bucal, favorecendo o desenvolvimento de infecções ${ }^{7,15,16}$.

Não existe, aparentemente, predisposição racial ou relacionada ao gênero para a MO induzida pela $\mathrm{QT}^{10}$.

\section{Fisiopatologia da MO}

A MO resulta de uma série de eventos biológicos que iniciam com um dano tecidual local e depende das condições de saúde bucal, da resposta imunológica e da predisposição genética dos pacientes. Os eventos biológicos surgem na submucosa e progridem em direção ao epitélio ${ }^{7,17}$.

O modelo biológico do desenvolvimento da mucosite é descrito em cinco fases ${ }^{18}$ :

- Fase de iniciação

- Fase de geração de resposta

- Fase de sinalização e amplificação

- Fase de ulceração e infecção

- Fase de reparação 
$\mathrm{Na}$ fase de iniciação, os agentes quimioterápicos e a radioterapia levam à geração de radicais livres e danos ao DNA, podendo resultar em morte celular, mas num número pequeno de células. Nessa fase, o dano ainda não causa lesão.

Na fase de geração de resposta, ocorre ativação do fator de transcrição nuclear KappaB (NFkB), aumento das citocinas inflamatórias como a Interleucina-1 $\beta$ (IL-1ß), a Interleucina 6 e fatores de necrose tumoral $\alpha$ (TNF- $\alpha$ ). A IL-1 $\beta$ inicia o processo inflamatório, determinando aumento da vascularização subepitelial e liberação de citocinas pró-inflamatórias pelo tecido conjuntivo. Ocorrem, em sequência, danos moleculares irreparáveis no ciclo celular e morte das células epiteliais basais por apoptose. Há um aumento da concentração dos agentes quimioterápicos no local devido ao aumento do fluxo sanguíneo. O TNF- $\alpha$ contribui causando dano tecidual.

$\mathrm{Na}$ fase de sinalização e amplificação, os mecanismos de respostas são ativados. TNF- $\alpha$ ativa as proteínas que contribuem para os danos celulares e teciduais, e o resultado é um eritema acentuado pela atrofia vascular e epitelial. Quatro a cinco dias após o início da QT, traumatismos pelas atividades diárias, como mastigar e falar, predispõem a ulceração.
A fase de ulceração e infecção coincide, comumente, com a fase de neutropenia, podendo as úlceras ser infectadas secundariamente e levar ao aumento da IL-1 $\beta$ e TNF- $\alpha$. Essa é a fase responsável pela dor da MO.

$\mathrm{Na}$ fase de reparação, ocorrem a proliferação celular e a reepitelização das áreas ulceradas. Sinalização da matriz extracelular induz as células epiteliais a migrarem através da pseudomembrana (fibrina) da úlcera, proliferando e cicatrizando a mucosa. A resolução da neutropenia e o controle das infecções também contribuem para a resolução das úlceras.

Apesar dos avanços no conhecimento do mecanismo da $\mathrm{MO}$, algumas questões referentes à relação entre a neutropenia e a MO necessitam ser compreendidas. Existem situações em que nem todos os pacientes com MO apresentam toxicidade hematológica.

\section{Classificação da MO}

A Organização Mundial de Saúde (OMS) classifica a MO em cinco categorias clínicas (Tab. 1).

Tabela 1 - Escala de toxicidade oral da OMS

\begin{tabular}{c|c|c|c|c}
\hline \multicolumn{3}{c|}{ Mucosite oral } & \multicolumn{2}{c}{ Mucosite oral grave } \\
\hline Grau 0 & Grau 1 & Grau 2 & Grau 3 & Ǵlceras. \\
\hline Sem alterações & Dor / eritema & Eritema e úlceras & Dieta exclusivamente líquida & $\begin{array}{c}\text { Não é possível a } \\
\text { alimentação }\end{array}$ \\
\hline
\end{tabular}
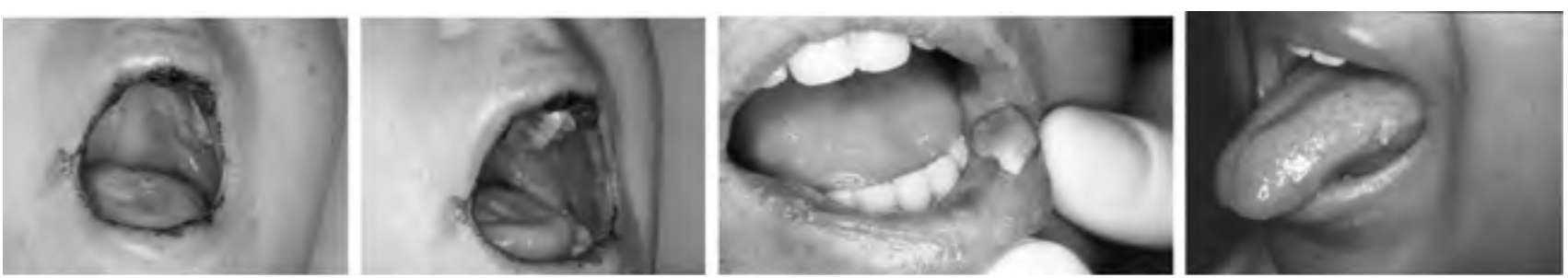

Figura 1 - Pacientes em tratamento com QT apresentando mucosite grau 3, presença de úlceras e dieta exclusivamente líquida

\section{Morbidade e mortalidade da MO}

A MO causa dor, restrição alimentar e determina rompimento da barreira fisiológica da mucosa, facilitando aquisição de infecção por microrganismos oportunistas. Nessas situações, frequentemente, é necessária a interrupção da terapia oncológica e hospitalização para utilização de antibióticos e analgésicos narcóticos endovenosos, representando desconforto ao paciente e aumento do custo do tratamento. Pacientes com MO e neutropenia apresentam risco relativo de septicemia quatro vezes maior que pacientes com neutropenia sem $\mathrm{MO}^{10}$.

Pacientes com doenças pulpares por cáries dentárias ou trauma, doença periodontal avançada ou infecções de baixo grau (associadas à erupção den- tária) apresentam risco maior de desenvolverem septicemia quando imunossuprimidos pela quimioterapia convencional ou utilizada na preparação para transplante de medula óssea ${ }^{19}$.

As duas doenças dentárias mais comuns, cárie e doença periodontal, associadas à ruptura da barreira de mucosa bucal observada durante o processo da MO, permitem a entrada de microrganismos nativos da cavidade bucal na corrente circulatória, podendo levar à septicemia ${ }^{4,7}$.

\section{Prevenção e tratamento da $\mathrm{MO}$}

A avaliação clínica odontológica de uma criança com câncer inicia com a análise das condições gerais do paciente, conhecimento da doença de base, 
doenças associadas e tipo de terapia antineoplásica a ser utilizada. Os cuidados odontológicos envolvem a avaliação da cavidade bucal desde o início do tratamento até o seu final, identificando vários aspectos e fatores para o desenvolvimento da mucosite.

A remoção de focos infecciosos através de procedimentos odontológicos cruentos ou não deve ser analisada e realizada levando-se em conta o melhor momento para a intervenção e a decisão do risco-benefício entre procedimento eletivo e emergencial ${ }^{4,5}$. A criação de um programa planejado, envolvendo limpeza dos dentes e bochechos com colutórios específicos para cada paciente, deve ser estimulada. Muitos estudos têm demonstrado a importância do cuidado bucal como auxílio na redução da $\mathrm{MO}$ e de infecções oportunistas, bem como na promoção do conforto ao paciente ${ }^{20}$. Próteses devem ser examinadas e ajustadas previamente para evitar traumas, e a sua higiene e descontaminação deve ser orientada. Aparelhos ortodônticos fixos devem ser removidos antes do início do tratamento com QT e/ou RT na região de cabeça e pescoço ${ }^{4,5}$. Restaurações metálicas podem causar efeito de interação com a irradiação em fenômeno conhecido como "retroespelhamento", produzindo irradiação secundária de baixa energia e dano à mucosa adjacente ${ }^{21}$.

Vários estudos têm sido realizados para estabelecer uma conduta profilática e terapêutica para a MO. No entanto, o tratamento ainda é essencialmente paliativo, baseado no uso de anestésicos tópicos, drogas anti-inflamatórias, agentes antimicrobianos tópicos e sistêmicos ${ }^{4,5,22}$.

As pesquisas com o laser de baixa intensidade surgiram em 1966 e são caracterizadas pela emissão de radiação de baixa intensidade, ou seja, não possuem efeito tecidual destrutivo ${ }^{23}$. Seus principais efeitos são a analgesia, a bioestimulação e a ação anti-inflamatória. Antunes et al. ${ }^{24}$ (2010) sugerem que a fotobiomodulação de baixa potência promove a cicatrização da mucosa bucal, reduz a inflamação, a dor e a intensidade, atuando na prevenção da severidade da $\mathrm{MO}$ em pacientes sob tratamento antineoplásico. Stokman et al. ${ }^{25}$ (2006) afirmam que alguns estudos mostraram que a ação do laser de baixa intensidade ocorre na fase IV, pertencente à patogênese da lesão, caracterizada pela fase de reparo tecidual por meio de células mesenquimais e sinalizadores da matriz extracelular. Entretanto, Eduardo et al. ${ }^{26}$ (2009) consideram que mais ensaios clínicos randomizados são necessários. Há ampla evidência de que o laser pode diminuir a dor e estabilizar as lesões na mucosa bucal, diminuir a progressão das lesões e acelerar sua cicatrização. Todavia, não há evidência sobre o real potencial terapêutico do laser na MO em pacientes em tratamento oncológico ${ }^{4,27}$.

No que se refere à prevenção, os principais agentes encontrados foram o gluconato de clorexidina a $0,12 \%$ em solução aquosa, combinação de antifúngicos e antibióticos associados a programas de cuidados bucais $^{28}$. Fatores de crescimento, benzidamina e medicações como a amifostina têm sido testados para a prevenção e o tratamento de mucosite, porém os resultados ainda não são conclusivos.

O gluconato de clorexidina a $0,12 \%$ é um antimicrobiano amplamente utilizado. Alguns trabalhos afirmam que, apesar de não impedir a ocorrência da mucosite, a clorexidina contribui para redução na severidade do quadro clínico, uma vez que os usuários da medicação apresentam graduações menores de MO. O gluconato de clorexidina é agente antimicrobiano tópico de liberação lenta que se liga à mucosa bucal, sugerido que a diminuição da gravidade da MO esteja vinculada à prevenção da contaminação secundária das lesões. A clorexidina apresenta uma substantividade, isto é, tempo de permanência ativa de aproximadamente 12 horas, exercendo uma ação bactericida inicial, imediatamente após o bochecho, combinada com uma ação bacteriostática prolongada ${ }^{29}$. A sua formulação em veículo aquoso, sem álcool ou conservantes, pode diminuir o desconforto durante o seu uso sem perda da eficácia ${ }^{30}$. Associações com algumas substâncias químicas como cálcio, detergentes aniônicos e flúor podem influenciar as ligações da clorexidina, reduzindo sua retenção e atividade antibacteriana ${ }^{29}$.

O aminoácido glutamina é uma importante fonte energética para os macrófagos, linfócitos e para as demais células do sistema imunológico. É considerada essencial em situações de hipercatabolismo associadas a estados de imunodeficiência frequentemente encontrados em pacientes oncológicos ${ }^{31}$. A L-Glutamina determina múltiplos efeitos no tecido mucoso. Tem sido relatada como eficaz e segura na redução da severidade da $\mathrm{MO}$, porém o número de ensaios clínicos ainda é limitado.

O palifermin é um fator de crescimento recombinante de queratinócitos (KGF) aprovado na Europa e recentemente nos Estados Unidos para a redução na incidência da MO em portadores de doenças hematológicas. Entretanto, em pacientes com tumores sólidos seus efeitos ainda não estão bem estabeleci$\operatorname{dos}^{21}$. Atua na medula óssea, aumentando a produção e mobilização de neutrófilos, embora não tenha revelado diferença estatisticamente significativa na redução da gravidade da $\mathrm{MO}^{32}$.

A amifostina é um citoprotetor antioxidante seletivo de amplo espectro. O princípio ativo desse fármaco atua nas diferenças fisiológicas entre as células normais e tumorais, que interferem no transporte seletivo do fármaco para dentro das células normais, protegendo-as. Entretanto, importantes efeitos colaterais são relatados, como náuseas, vômitos e hipotensão ${ }^{20}$.

O sucralfato é um fármaco à base de sulfato básico de alumínio e sacarose, amplamente utilizado para proteção da mucosa gástrica, pois se liga fortemente às proteínas na mucosa inflamada ou exsudato de úlceras, formando uma camada que protege as lesões. Tem sido usado para auxiliar no tratamento da $\mathrm{MO}^{33}$. 
A benzidamina é uma droga não esteroide, com propriedades anestésica, analgésica, anti-inflamatória e antimicrobiana. Estudos têm demonstrado que ela pode inibir a produção e os efeitos das citocinas pró-inflamatórias, principalmente o TNF-alfa ${ }^{22,34}$.

A crioterapia envolve a dissolução de fragmentos de gelo na cavidade bucal por 5 minutos antes e 25 minutos após a administração de quimioterápicos. A intenção é minimizar o efeito citotóxico do quimioterápico sobre a mucosa pela diminuição da circulação sanguínea durante os elevados picos do quimioterápico no sangue. A utilização desse mecanismo é simples e isenta de efeitos adversos, apresentando bons resultados ${ }^{5,35,36}$.

$\mathrm{O}$ concentrado bioaderente bucal em gel indicado para o tratamento e alívio de dor associado à mucosite oral tem sido avaliado, e as evidências iniciais indicam que pode proporcionar alívio rápido e durável da dor, pois forma uma barreira aderente sobre a mucosa bucal ${ }^{37-39}$. Lactoperoxidase e glicose oxidase geram um fluxo constante de íons hipotiocianato $\left(\mathrm{OSCN}^{-}\right)$e ácido hipotiocianoso (HOSCN), que são fortes agentes antimicrobianos. Lisozima é uma enzima natural que hidrolisa a parede celular bacteriana, e a lactoferrina liga-se ao ferro inviabilizando seu acesso à bactéria. O sistema de enzimas ajuda a inibir o crescimento de bactérias patogênicas mantendo a umidade bucal por até 6 horas.

\section{Considerações finais}

A MO é uma importante e frequente intercorrência observada durante o tratamento oncológico, e sua prevenção e tratamento precoce auxiliam a minimizar esse efeito colateral da QT e/ou RT. As infecções oportunistas iniciadas na cavidade oral, a dificuldade para alimentação e a neutropenia aumentam o risco de septicemia.

Existem algumas sugestões de tratamento paliativo da MO. A inclusão do gluconato de clorexidina na forma de bochechos em solução aquosa auxilia na prevenção de infecções secundárias sobre as lesões da MO. A utilização de laserterapia de baixa intensidade também tem sido recomendada para alívio dos sintomas desses pacientes.

A inclusão da avaliação odontológica das crianças com câncer no planejamento do tratamento quimioterápico e/ou radioterápico na região cérvico-facial auxiliará na prevenção de complicações que possam inviabilizar o seguimento adequado do tratamento proposto.

\section{Abstract}

Oral mucositis (OM) is characterized by inflammation of the oral mucosa, and it is frequently observed after chemotherapy and radiotherapy in the head and neck regions. It ranges from localized erythema to extensive ul- cers, and it may be aggravated by inadequate oral health. Objective: this study aims to conduct a literature review on OM. Literature review: oral mucositis represents a high risk of infection, which may progress to severe systemic infections interfering with treatment and patient survival. The knowledge of etiology and mechanisms of prevention and treatment are important to reduce the risks associated with opportunistic infections, feeding impairments, and septicemia. Oral mucositis is a complex and multifactorial process involving all tissues and cellular elements of the oral mucosa. New evidences suggest that the risk of developing oral mucositis may be influenced by genetic factors. The approaches have been constantly evaluated seeking prevention, treatment, reduced risks, and improved quality of life for patients. Final considerations: previous adequate oral health status, and dental care follow-up during cancer treatment are important in preventing stomatological complications. Preventive and palliative treatment of $O M$ helps in preventing secondary infections, and it is recommended for symptoms relief.

Keywords: Oral mucositis. Chemotherapy. Radiotherapy. Oral health

\section{Referências}

1. Zanin T. Laser Diodo $660 \mathrm{~nm}$ na prevenção e tratamento da mucosite oral em humanos induzida por radioterapia e/ ou quimioterapia [Dissertação de Mestrado]. São José dos Campos: Engenharia Biomédica Universidade do Vale do Paraiba; 2006.

2. Junqueira LC, Carneiro J. Histologia básica. Rio de Janeiro: Guanabara Koogan; 2008.

3. Neville BW, Damm DD, Allen CM, Bouquot JE. Patologia oral e maxilofacial. 3. ed. Rio de Janeiro: Guanabara Koogan; 2009.

4. Santos PSS, Soares Jr LAV. Medicina bucal: a prática na odontologia hospitalar. São Paulo: Santos; 2012

5. Santos PSS, Messaggi AC, Mantesso A, Magalhães MHCG. Mucosite oral: perspectivas atuais na prevenção e tratamento. RGO 2009; 57(3):339-44.

6. Albuquerque RA, Morais VL, Sobral APV. Protocolo de atendimento odontológico a pacientes oncológicos pediátricos revisão de literatura. Rev Odontol Unesp 2007; 36(3):275-80.

7. Volpato LER, Silva TC, Oliveira TM, Sakai VT, Machado MAAM. Mucosite bucal radio e quimioinduzida. Rev Bras Otorrinolaringol 2007; 73(4):562-8.

8. Trotti A, Bellm LA, Epstein JB, Frame D, Fuchs HJ, Gwede $\mathrm{CK}$, et al. Mucositis incidence, severity and associated outcomes in patients with head and neck cancer receiving radiotherapy with or without chemotherapy: a systematic literature review. Radiother Oncol 2003; 66(3):253-62.

9. Epstein JB, Schubert MM. Oropharyngeal mucosites in cancer therapy. Review of pathogenesis, diagnosis and management. Oncoloy (Williston Park) 2003; 17:1767.

10. Treister NS, Woo SB, Cruz Jr PD, Butler DF, Eisen D, Crawford JH, et al. Chemotherapy-Induced Oral Mucositis Treatment \& Management [periódico online] 2013 [citado 2013 Jul 4]. Disponível em URL: http://www.emedicine.medscape.com/ article/1079570-treatment.

11. Bonan PRF, Lopes MA, Alves FA, Almeida OP. Aspectos clínicos, biológicos, histopatológicos e tratamentos propostos para a mucosite oral induzida por radioterapia: revisão de literatura. Rev Bras Cancerol 2005; 51(3):235-42. 
12. Sonis ST. Mucositis as a biological process: a new hypothesis for the development of chemotherapy-induced stomatotoxicity. Oral Oncol 1998; 34:39-43.

13. Turthal NS, Erdal S, Karacay S. Efficacy of treatment to relieve mucositis-induced discomfort. Support Care Cancer [periódico online] 2000 [citado 2013 Mar 27]; 8:55-8. Disponível em URL: http//www.ncbi.nlm.nih.gov/pub$\mathrm{med} / 10650899$.

14. McCarthy GM, Awde JD, Ghandi H, Vincent M, Kocha WI. Risk factors associated with mucositis in cancer patients receiving 5-fluorouracil. Oral Oncology [periódico online] 1998 [citado $2013 \mathrm{Fev}$ 5]; 34:484-90. Disponível em URL: http://www.sciencedirect.com/science/article/pii/ S1368837598000682.

15. Adamietz IA, Rahn R, Böttcher HD, Schäfer V, Reimer K, Fleischer W. et al. Prophylaxis with povidone-iodine against induction of oral mucositis by radiochemoterapy. Support Care Cancer 1998; 6:373-7.

16. Chan CWH, Chang AM, Molassiotis A, Lee IYM, Lee GCT. Oral complications in Chinese cancer patients undergoing chemotherapy. Support Care Cancer [periódico online] 2003 [citado 2013 Abr 19]; 11:48-55. Disponível em URL: http:// link.springer.com/article/10.1007/s00520-002-0413-9.

17. Sonis ST, O Donnell KE, Popat R, Bragdom C, Phelan S, Cock $\mathrm{D}$, et al. The relationship between mucosal cyclooxygenase-2 (COX-2) expression and experimental radiationinduced mucositis. Oral Oncology 2004; 40:170-6.

18. Sonis ST. The Pathobiology of mucositis. Nature Reviews Cancer 2004; 4(4):277-84.

19. Reuscher TJ, Sodeifi A, Scrivani SJ, Kaban L, Sonis ST. The impact of mucositis on alpha-hemolytic streptococcal infection in patients undergoing autologous bone marrow transplantation for hematologic malignancies. Cancer [periódico online] 1998 [citado 2013 Mar 24]; 82(11):2275-81. Disponível em URL: http://www.ncbi.nlm.nih.gov/pubmed/9610710.

20. Souza CA, Vigorito AC, Aranha FGP, Oliveira GB, Eid KAB, Ruiz MA. Terapêutica citoprotetora em pacientes tratados com quimio e/ou radioterapia anti neoplásica. Rev Bras Hematol Hemoterap 2000; 22(2):123-8.

21. Hanriot RM, Mello ALS. Atualização da prevenção e manejo da mucosite radioinduzida em câncer de cabeça e pescoço. Prática Hospitalar 2008; 10(60):106-10.

22. Rubenstein EB, Peterson DE, Schubert M, Keefe D, McGuire D, Epstein J, et al. Clinical practice guidelines for the prevention and treatment of cancer therapy - induced oral and gastrointestinal mucositis. Willey InterScience [periódico online] 2004 [citado 2013 Abr 14]; 100(9):2026-46. American Cancer Society. Disponível em URL: http://www. ncbi.nlm.nih.gov/pubmed/15108223.

23. Rozza RE. Avaliação de diferentes estratégias para prevenção de mucosite bucal em pacientes sob quimioterapia antineoplásica - ensaio clínico randomizado [Dissertação de Mestrado]. Paraná: PUCPR; 2011.

24. Antunes HS, de Azevedo AM, da Silva Bouzas LF, Adão CA, Pinheiro CT, Mayhe R, et al. Law-power laser in the prevention of induced oral mucositis in bone marrow transplantation patients: a randomized trial. Blood 2007; 109(5):2250-5.

25. Stokman MA, Spijkervet FKL, Boezen HM, Schouten JP, Roodenburg JLN, de Vries EGE. Prevention intervention possibilities in radiotherapy-and chemotherapy-induced oral mucositis: results of meta-analyses. J Dental Research 2006; 85(8):690-700.

26. Eduardo FP, Bezinelli L, Luiz AC, Correa L, Vogel C, Eduardo CP. Severity of oral mucositis in patients undergoing hematopoietic cell transplantation and oral laser phototherapy protocol: a survey of 30 patients. Photomed Laser Surg 2009; 27(1):137-44.

27. Schubert MM, Eduardo FP, Guthrie KA, Franquin JC, Bensadoun RJ, Migliorati CA, et al. A phase III randomized doble-blind placebo-controled clinical trial to determine the efficacy of low level laser therapy for the prevention of oral mucositis in patients undergoing hematopoietic cell transplantation. Suport Care Cancer 2007; 15(10):1145-54.

28. Sandoval RL, Koga DH, Buloto LS, Suzuki R, Dib LL. Management of chemo and radiotherapy induced oral mucositis with low-energy laser: initial results of A.C. Camargo Hospital. Applied Oral Science 2003; 11(4):337-41.

29. Zanatta FB, Rosing CK. Clorexidina: mecanismo de ação e evidências atuais de sua eficácia no contexto do biofilme supragengival. Scientific 2007; 1(2):35-43.

30. Olsson H, Asklow B, Johansson E, Slote C. Rinsing with alcohol-free or alcohol-based chlorhexidine solutions after periodontal surgery. A double-blind, randomized, cross-over, pilot study. Sweed Dent J 2012; 36(2):91-9.

31. Boligon CS, Huth A. O impacto do uso de glutamina em pacientes com tumores de cabeça e pescoço em tratamento radioterápico e quimioterápico. Rev Bras Cancerologia 2011; 57(1):31-8.

32. Ferreira P, Gamba MA, Saconato H, Gutierrez MGV. Tratamento da mucosite em pacientes submetidos a transplante de medula óssea: uma revisão sistemática. Acta Paul Enferm 2011; 24(4):563-70.

33. Ulcermin - Jaba. Disponível em URL: http:// www.jaba. pt/_Media/.../882cf519-a163-45c7-aac2-fcc0946e113b.pdf. Acesso em: Ago 2013

34. Assunção Jr JNR, Raitz R, Cavalcanti BN, Teixeira VP. Uso de colutórios na prevenção e tratamento da mucosite oral. Rev Bras Ciênc Saúde 2006; 8:6-11.

35. Migliorati CA, Oberle-Edwards L, Schubert MM. The role of alternative and natural agents, cryotherapy and/or laser for management of alimentary mucositis. Support Care Cancer 2006; 14(6):533-40.

36. Kroetz FM, Czlusniak GD. Alterações bucais e condutas terapêuticas em pacientes infanto-juvenis submetidos a tratamentos anti-neoplásicos. Publ UEPG Biol Health Sci 2003; 9(2):41-8.

37. Kuhn A, Porto FA, Miraglia P, Brunetto AL. Low-level infrared laser therapy in chemoterapy-induced oral mucositis. J Pediatr Hematol Oncol 2009; 31(1):33-7.

38. Negrin RS, Bedard JF, Toljanic JA. Oral toxicity associated with chemotherapy. Published online UpToDate 2012 [citado 2013 Jun 24]. Disponível em URL: http://www.uptodate. com/contents/oral-toxicity-associated-with-chemotherapy.

39. Keefe DM, Schubert MM, Elting LS, Sonis ST, Epstein JB, Raber-Durlacher JE. Diretrizes de prática clínica para a prevenção e tratamento da mucosite. Câncer 2007; 109:820-31.

\section{Endereço para correspondência:}

Isabel Nemoto Vergara Sasada

Unidade de Odontologia do Hospital de Clínicas de Porto Alegre, UFRGS

Rua Ramiro Barcelos, 2350 - Largo Eduardo Faraco 90035-903 Porto Alegre - RS

Fone: (51) 81247238

E-mail: isasada@hcpa.ufrgs.br

Recebido: 11/07/2013. Aceito: 24/09/2013. 